\title{
A PROSPECTIVE STUDY OF THE MANAGEMENT OF OPEN FRACTURES OF TIBIA
}

\author{
Abul Kalam Mohammad, Mahesh Shrivastav, Pradeep Gupta, \\ and Awais Sayed
}

\begin{abstract}
:
A Prospective study of 30 cases of type III open fractures of tibia is conducted at Nepal Medical College, Kathmandu and Nobel Medical College, Biratnagar from 2009 to 2011. All cases included in the study were initially managed by wound debridement and external fixation, and followed-up regularly for 10 months for outcome. Five cases developed deep infection and 10 had superficial infection, which subsided with prolonged use of antibiotics. Union was obtained in 7to 8 months for 28 cases. Two cases resulted in non- union which had been managed by bone grafting. Open Type III fractures of tibia are still a difficult and challenging problem for orthopedic surgeons. Almost all cases are required for twice surgery including secondary closure, skin grafting and readjustment.
\end{abstract}

Key words: Open tibial fractures, External fixator.

\section{Introduction:}

Open Type III fractures of tibia are very common due to road traffic accidents. It can be life threatening injuries and can cause severe permanent disability. Decision making on preferred treatment is complicated due to higher energy injury. External fixation is accepted by most orthopedic surgeons as the treatment of choice for Type III open tibial fractures because of its easiness for prolonged period of dressing, early stabilization and reconstruction of soft tissue envelop ${ }^{1,2,3}$.

\section{Material and methods:}

A prospective study of all consecutive patients over the period of study, 2009-2011, fulfilling the inclusion criteria were enrolled in the study. Those who were below 12 years of age and were suffering from diabetes mellitus were excluded from the study. Similarly cases who did not give the consent and were unable to complete their follow-up were also excluded from the study.
All subjects enrolled in the study were treated under C-arm mobile image intensifier by early and proper wound toilet and the application of AO type external fixator and were maintained on IV antibiotics, regular dressing and early mobilization. Patients were mobilized as soon after adequate soft tissue healing. The patients were non-weight bearing until the adequate bone union. Bone grafting was done within first four months. The duration of external fixator was determined by the operative surgeons, based on the state of the soft tissues and the extent of bone union. Sometimes the patients desire to have the fixator removed.

\section{Results:}

A total number of 40 patients with type III open tibial fractures were initially enrolled in the study. They were treated with external skeletal fixation. Out of 40 cases initially enrolled, 06 cases absconded and did not appear in their follow-up visits; similarly 04 cases were also referred to plastic surgeon for management of skin problems. 
Out of 30 cases, who completed the study, 10 of the cases were male (33.33\%) and 20 were female $(66.66 \%)$, with mean age near about 30.5 years.

There were 16 Type III A fractures with an average time to union of 28 weeks. The average period of external fixation was 14 weeks (12-16 weeks).The average hospitalization time was about two weeks and to work was at an average of 36 weeks after the fracture.

There were 12 Type III B fractures with an average time to union of 32 weeks. Of these 4 patients had extensive osteomyelitis and 6 had superficial wound. 2 patients who had extensive wound infection had to undergo secondary procedures like bone grafting and skin care. 6 patients had pin track infection
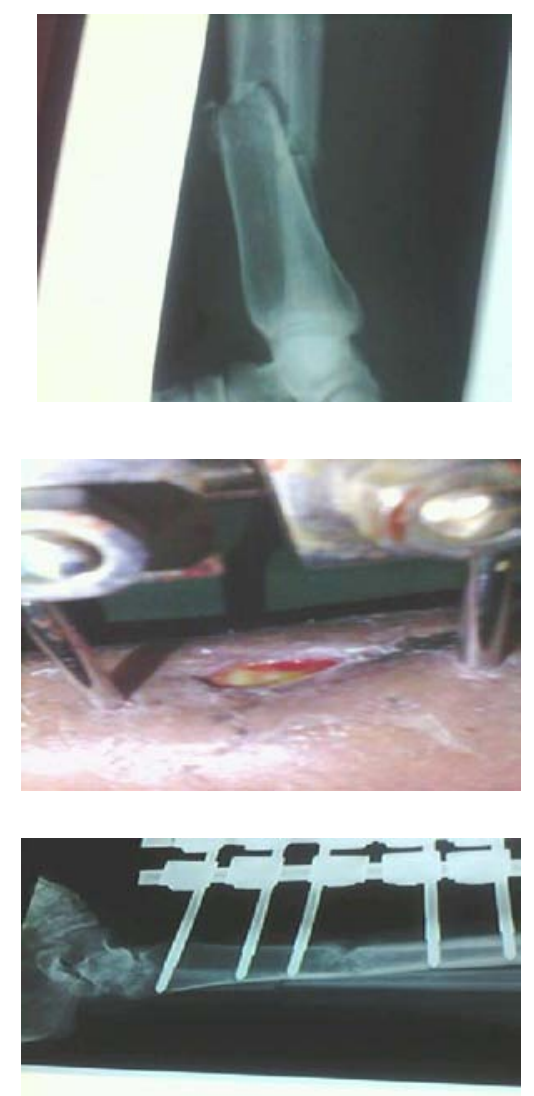

and losing of pins and in these patients the external fixator was readjusted. Two $(16.6 \%)$ patients developed nonunion and were readmitted for other procedures. Of the fractures that united 10 had an acceptable position giving over all valgus rates of $20 \%$.

The average hospitalization time was one month. Most of the patients returned to work after 14-15 month of surgery.

There were 2 Type III $\mathrm{C}$ fractures with an average time of union about 40 weeks. Both of them undergone multiple surgical procedures.

In our study some of the patients had fixator removed at an early stage because of psychiatric problems and was placed in a patellar tendon bearing cast until union had completed
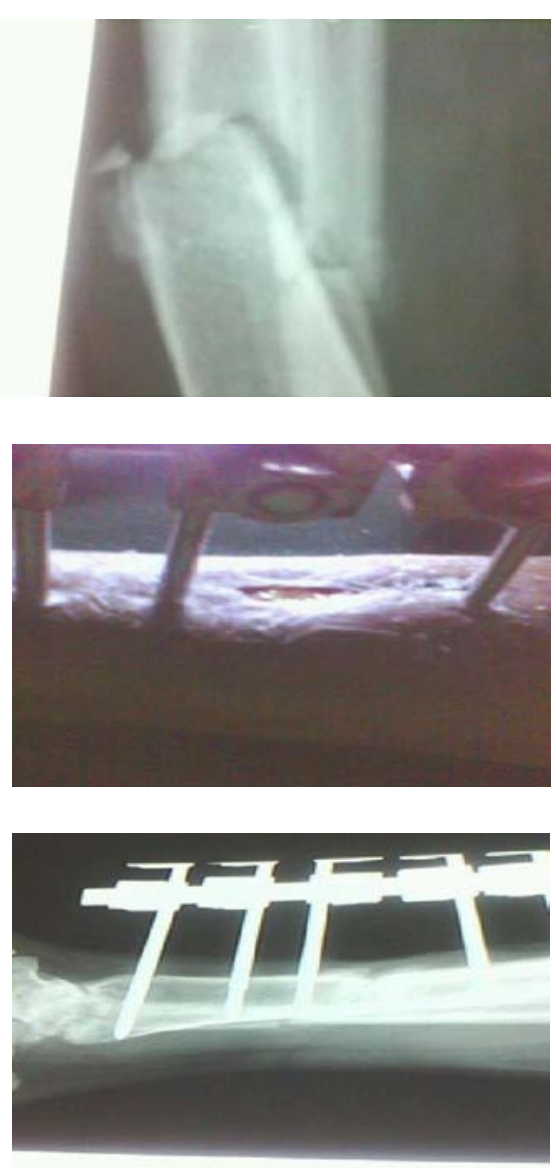


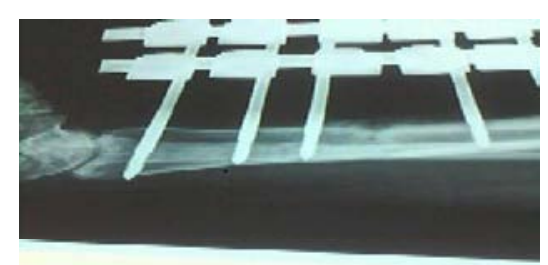

\section{Discussion:}

Our results is in consistent with some of other studies that used external fixation in Type III open fractures ${ }^{4,5,6 .}$ We found excellent result for open tibial fractures treated with external fixator system. Orthopaedic surgeon treating open fractures of tibia by external fixator system because of strong gripping of fracture sites. So this system controls the movement and rotation of fracture sites as well as very easy for proper dressing. A previous reviewed had shown the poor results if the fixator were removed earlier ${ }^{7,8}$.

In our study mean time of union is 33.3 weeks. Chan et al (1984) reported $41 \%$ incidence of malunion and $62 \%$ incidence of joint stiffness. $^{4}$ The results for the different fractures subtypes highlight the importance of classifying these fractures adequately and suggest that the criteria on which Gustile et al based there sub-classification of type III fractures are appropriate ${ }^{9}$

It is clear that each of the type III subtypes carries a very different prognosis. Type III A fractures are associated with a low incidence of infection and a relatively excellent functional result, with earlier return to Type III B fractures carry a significantly not so good prognosis, not only in the time to union but in the marked increased in the incidence of osteomyelitis. The hospitalization time for

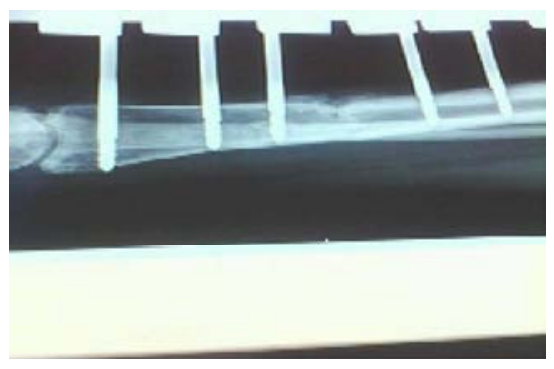

type III B fractures in our series averaged 40 days. Return to work was delayed. Prognoses for type IIIC fractures are poor.

Generally open type III fractures which were not reduced properly at the time of initial reduction but in our study image intensifier used and try to fracture sites reduced almost in proper position. Good results depend on good surgical technique and early skin cover. We found excellent result for open tibial fractures treated with external fixator system. Orthopaedic surgeon treating open fractures of tibia by external fixator system because of strong gripping of fracture sites ${ }^{10,11}$. So this system controls the movement and rotation of fracture sites as well as very easy for proper dressing

\section{Conclusion:}

External Fixation Systems of open type III fractures of tibia is a gold standard method of treatment with advantages of low rate of wound infection and early mobilization.

\section{References:}

1. Behrens F, and Searls K. External fixation of tibia: basic concept and prospective Valuation. J.B.J.S:1986; 68 B: 264-54.

2. Burgess et al. Management of type III open tibial fractures. orthop clin am 1987; 18:85-93.

3. Caudle Rj, and Stern PJ. Open fractures of tibia J.B.J.S:1987; 69-S:801-7 
4. Chan et al. Management of type III open tibial fractures. Injury, 194; 16:157-65.

5. Chapman M.W. Role of intramedullary fixation in open fractures. Clin orthrop 1986; 212:26-34.

6. Court et al. External fixator in management

7. Gustilo RB and Anderson JT. Prevention of infection in long bone fractures. J.B.J.S, 1976; 58-A: 453-8.
8. Karlstorm G, Olerud. External fixator in ioen tibial fractures.J.B.J.S.1980; 62-A:1264-73

9. Lange et al. Open tibial fractures with associated vascular injuries: Prognosis for limb salvage .J. Trauma 1985;25:203-8

10. Lawyer et al. External fixation in open tibial fracture J.B.J.S 1980; 62-A: 1264-73.

11. Velazco A, and Fleming LL. Open tibial fractures treated by external fixation. Clin Orthop $1983 ; 180: 125-32$

of tibial fractures. JR soc Med 1985; 78:830-7

Correspondence Address: Dr. Abul Kalam Mohammad, Department of Orthopedics, Nobel Medical College and Teaching Hospital, Kanchanbari-5, Bitatnagar. 